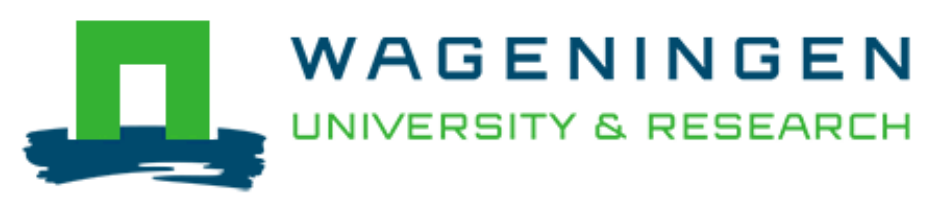

\title{
Diabetes and necrotizing soft tissue infections-A prospective observational cohort study: Statistical analysis plan
}
Rosén, A., Arnell, P., Madsen, M. B., Nedrebø, B. G., Norrby-Teglund, A., Hyldegaard, O., ... Skrede, S.

This article is made publically available in the institutional repository of Wageningen University and Research, under article 25fa of the Dutch Copyright Act, also known as the Amendment Taverne.

Article $25 \mathrm{fa}$ states that the author of a short scientific work funded either wholly or partially by Dutch public funds is entitled to make that work publicly available for no consideration following a reasonable period of time after the work was first published, provided that clear reference is made to the source of the first publication of the work.

For questions regarding the public availability of this article, please contact openscience.library@wur.nl.

Please cite this publication as follows:

Rosén, A., Arnell, P., Madsen, M. B., Nedrebø, B. G., Norrby-Teglund, A., Hyldegaard, O., ... Skrede, S. (2018). Diabetes and necrotizing soft tissue infections-A prospective observational cohort study: Statistical analysis plan. Acta Anaesthesiologica Scandinavica, 62(8), 1171-1177.

https://doi.org/10.1111/aas.13130 


\title{
Diabetes and necrotizing soft tissue infections-A prospective observational cohort study: Statistical analysis plan
}

\author{
A. Rosén $^{1}$ (D) | P. Arnell ${ }^{1}$ | M. B. Madsen² ${ }^{2}$ | B. G. Nedrebø $\varnothing^{3,4}$ | A. Norrby-Teglund ${ }^{5}$ | \\ O. Hyldegaard ${ }^{6}$ | V. M. dos Santos ${ }^{7,8}$ | F. Bergey ${ }^{7}$ | E. Saccenti ${ }^{8}$ | \\ INFECT Study Group ${ }^{\dagger}$ | S. Skrede ${ }^{4,9}$
}

${ }^{1}$ Department of Anaesthesia and Intensive Care Medicine, Sahlgrenska University Hospital/Ostra, Gothenburg, Sweden

${ }^{2}$ Department of Intensive Care,

Copenhagen University Hospital, Rigshospitalet, Copenhagen, Denmark

${ }^{3}$ Department of Medicine, Haugesund County Hospital, Haugesund, Norway

${ }^{4}$ Department of Clinical Science, University of Bergen, Bergen, Norway

${ }^{5}$ Centre for Infectious Medicine, Karolinska Institutet, Karolinska University Hospital, Stockholm, Sweden

${ }^{6}$ Department of Anaesthesia, Center of Head and Orthopaedics, Copenhagen University Hospital, Rigshospitalet,

Copenhagen, Denmark

${ }^{7}$ LifeGlimmer GmbH, Berlin, Germany

${ }^{8}$ Laboratory of Systems and Synthetic Biology, Wageningen University \& Research, Wageningen, The Netherlands

${ }^{9}$ Department of Medicine, Haukeland University Hospital, Bergen, Norway

Correspondence

A. Rosén, Department of Anaesthesia and Intensive Care Medicine, Sahlgrenska University Hospital/Ostra, Gothenburg, Sweden.

Email: anders.rosen@vgregion.se

Funding information

INFECT is funded by the European Union Framework Programme 7 under the grant agreement 305340.
Background: Necrotizing soft tissue infections (NSTIs) are rare but carry a high morbidity and mortality. The multicenter INFECT project aims to improve the understanding of the pathogenesis, clinical characteristics, diagnosis, and prognosis of NSTIs. This article describes the study outline and statistical analyses that will be used.

Methods: Within the framework of INFECT project, patients with NSTI at 5 Scandinavian hospitals are enrolled in a prospective observational cohort study. The goal is to evaluate outcome and characteristics for patients with NSTI and diabetes compared to patients with NSTI without diabetes. The primary outcome is mortality at 90 days after inclusion. Secondary outcomes include days alive and out of ICU and hospital, SAPS II, SOFA score, infectious etiology, amputation, affected body area, and renal replacement therapy. Comparison in mortality between patients with diabetes type 1 and 2 as well as between insulin-treated and non-insulin-treated diabetes patients will be made. Clinical data for diabetic patients with NSTI will be reported. Conclusion: The study will provide important data on patients with NSTI and diabetes.

\section{1 | INTRODUCTION}

\section{1 | Necrotizing soft tissue infections}

Necrotizing soft tissue infections (NSTIs) are uncommon, severe, and potentially lethal conditions. ${ }^{1,2}$ Surviving often comes at the prize of

${ }^{\dagger}$ INFECT Study Group members are in Appendix 1 life-lasting morbidity. ${ }^{3}$ NSTI typically presents with pain, swelling, and skin discoloration ${ }^{4}$. The infection may involve any layer of soft tissue, causing local ischemia, tissue destruction, and necrosis. ${ }^{4-6}$ Bacterial spread proceeds swiftly, frequently along fascial planes. Host immunological response results in systemic toxicity. Shock, organ failure, and eventually death may result. ${ }^{7-9}$

Microbiological findings frequently exhibit a distinguishing pattern with polymicrobial growth in samples from the pelvic region ${ }^{10,11}$ 
and monomicrobial growth when a limb is affected. In the latter case, group A streptococcus is often found in cultures, but many other bacterial findings have been described as well. ${ }^{4,12-14}$

Mainstays of successful treatment are aggressive resuscitation, intravenous antibiotics, and prompt surgical resection of affected tissue. ${ }^{4,6,8,10,12}$ Hyperbaric oxygen treatment (HBOT) is, by some, considered an adjuvant treatment resource. ${ }^{8,15,16}$ Due to concurrent circulatory, respiratory, and renal disturbances, most patients are treated at intensive care units (ICU).

Diabetes is associated with an increased risk of infections $s^{4,17,18}$ and is overrepresented among patients with NSTI, with a reported frequency of $20 \%-70 \% .^{10,11,15,19-23}$ In contrast, the World Health Organization (WHO) estimates global diabetes prevalence to be $8.5 \%$ in adults. ${ }^{24}$ Concomitant diabetes seems to be a negative prognostic factor among patients with NSTI with increased risk of death, amputation, and infectious complications. ${ }^{22,23,25-27}$ Hyperglycemia could be a factor associated with outcome in diabetic patients with NSTI. ${ }^{26}$

\section{2 | Hypothesis}

Our research hypothesis is that there are significant differences in both clinical characteristics, bacterial findings, and outcome among patients with NSTI and diabetes compared to patients with NSTI without diabetes.

\section{3 | Study aim}

The aim of this study is to report outcome for patients with NSTI and diabetes compared to patients with NSTI without diabetes and to report clinical data for patients with NSTI and diabetes.

\section{2 | METHODS}

\section{1 | Study design}

Prospective observational cohort study.

\subsection{Population}

Adult $(\geq 18$ years) patients with NSTI.

\section{3 | Exposure group}

Patients with diabetes type 1 or type 2 .

\subsection{Comparison group}

Patients without diabetes type 1 or type 2 .

\subsection{Outcome}

\subsection{1 | Primary outcome}

1. All-cause mortality 90 days after inclusion.

\subsubsection{Secondary outcomes}

1. Days alive and out of ICU at day 30 after inclusion.

2. Days alive and out of hospital at day 90 after inclusion.

3. Amputation of the extremities during ICU stay. Site of amputation is defined as either upper arm, lower arm, hand, finger, upper leg, lower leg, foot, toe, or penis.

4. Sequential organ failure assessment score (SOFA score) day 1 in the ICU.

SOFA score is used to measure severity of disease. It is based on the assessed function of respiratory, nervous, and cardiovascular systems, liver, kidneys, and coagulation. ${ }^{28,29}$

5. SAPS II score at ICU admission.

SAPS II score is used to measure severity of disease at ICU admission. It is based on measured physiological parameters and results from blood samples, previous health status, and information about the ICU admission. ${ }^{30}$

6. The presence of monomicrobial growth of group A streptococci (GAS) in cultures.

Tissue and blood samples taken at referring hospital and study hospital are used. ${ }^{31}$

7. The presence of monomicrobial growth of group B streptococci in cultures.

Tissue and blood samples taken at both referring hospital and study hospital are used. ${ }^{31}$

8. The presence of monomicrobial growth of Staphylococcus aureus in cultures.

Tissue and blood samples taken at both referring hospital and study hospital are used. ${ }^{31}$

9. The presence of polymicrobial flora in cultures.

Tissue and blood samples taken at both referring hospital and study hospital are used. ${ }^{31}$

10. Affected body area at inclusion.

Affected body area is defined as either head and neck, upper body (including upper extremities), abdomen and anogenital region, or lower extremities.

11. Any renal replacement therapy (RRT) within 90 days after inclusion.

RRT is defined as either intermittent hemodialysis or continuous renal replacement therapy.

\subsection{3 | Exploratory outcomes}

Comparisons are made within the group of patients with diabetes type 1 and type 2 :

1. Association between glycated hemoglobin (A1C) values before admission and all-cause mortality 90 days after inclusion.

2. All-cause mortality 90 days after inclusion in patients with diabetes type 1 compared to patients with diabetes type 2 .

3. All-cause mortality 90 days after inclusion in patients with insulin-treated diabetes (type 1 and type 2) compared to non-insulintreated patients with diabetes type 2 . 
4. Association between $\mathrm{A} 1 \mathrm{C}$ values before admission and days alive and out of ICU at day 30 after inclusion.

5. Association between $\mathrm{A} 1 \mathrm{C}$ values before admission and the presence of polymicrobial flora in cultures.

Tissue and blood samples taken at both referring hospital and study hospital are used. ${ }^{31}$

6. Association between $\mathrm{A} 1 \mathrm{C}$ values before admission and affected body area at inclusion.

Affected body area is defined as either head and neck, upper body (including upper extremities), abdomen and anogenital region, or lower extremities.

7. Association between $\mathrm{A} 1 \mathrm{C}$ values before inclusion and any RRT within 90 days after inclusion.

RRT is defined as either intermittent hemodialysis or continuous renal replacement therapy.

\subsection{4 | Reported clinical data, comorbidities, risk factors for NSTI, and medication}

-Sex;

-Age;

-Weight (before surgery; if not available then best estimate);

-Body mass index;

-Smoking habits (currently smoking/estimated package years);

-AIDS (HIV positive with clinical complications as Pneumocystis jiroveci pneumonia, Kaposi's sarcoma, lymphoma, tuberculosis, or toxoplasma infection);

-Other immunodeficiencies;

-Active malignancy;

-Metastatic carcinoma (proven metastasis by surgery, CT scan, or any other method);

-Hematologic cancer;

-Chronic obstructive pulmonary disease (COPD) or asthma;

-Current or previous cardiovascular disease (hypertension, myocardial infarction, angina pectoris, heart failure, apoplexy);

-Peripheral vascular disease;

-Diabetes type 1;

-Non-insulin-treated diabetes type 2;

-Insulin-treated diabetes type 2;

-Chronic kidney disease (s-creatinine $>100$ before admission for NSTI or any kind of dialysis);

-Chronic liver disease (any history of chronic liver disease);

-Varicella;

-Rheumatoid disease;

-Previously admitted for necrotizing soft tissue infection;

-Surgery within the previous 4 weeks;

-Blunt trauma (a hit by a blunt object or a fall within the 4 weeks prior to diagnosis);

-Penetrating trauma (a hit by a sharp object penetrating the skin within the 4 weeks prior to diagnosis);

-Chronic wound or other skin disease;

-Intravenous drug use;
-Immunosuppressive drugs given for rheumatoid disease or malignant disease;

-Steroids.

\section{6 | The INFECT project}

Improving Outcome of Necrotizing Fasciitis: Elucidation of Complex Host and Pathogen Signatures that Dictate Severity of Tissue Infection (INFECT) is an international, prospective, multicenter observational research project. The goal is to improve the understanding of the pathogenesis, clinical characteristics, diagnosis, and prognosis of NSTIs. ${ }^{32}$ Within the framework of INFECT, patients are recruited from 5 Scandinavian hospitals which are all referral hospitals for patients with NSTI.

INFECT is registered at ClinicalTrials.gov (NCT01790698).

\subsection{Study setting}

Patients are recruited from intensive care departments in the following hospitals:

Copenhagen University Hospital, Rigshospitalet, Copenhagen, Denmark.

Sahlgrenska University Hospital/Ostra, Gothenburg, Sweden.

Karolinska University Hospital, Stockholm, Sweden.

Blekinge County Council Hospital, Karlskrona, Sweden.

Haukeland University Hospital, Bergen, Norway.

\subsection{Eligibility}

Patients arriving at a study hospital during the study period with a suspected NSTI are eligible for inclusion.

\section{9 | Inclusion criteria}

Adult ( $\geq 18$ years) patients with confirmed NSTI treated in one of the participating hospitals who consent to participate in the study.

The diagnosis of NSTI is made by the surgeon during the primary operation. It is based on, but not restricted to, findings of necrosis, dissolved or deliquescent soft tissue, and fascial and muscular affection.

\subsection{Exclusion criteria}

Denial or withdrawal of consent.

Later refuted NSTI diagnosis.

\subsection{1 | Subject enrollment}

Patients are included by local research staff at each hospital. Consent is obtained according to national ethical regulations.

The research staff ensures that the consenting party is given oral and written information approved by the respective ethical committee about the nature, purpose, possible risk, and benefit of the study. 


\subsection{Group allocation (exposure/comparison)}

Patients with an existing diagnosis of diabetes type 1 or type 2 in the medical records before inclusion in the study are allocated to the exposure group.

Patients without an existing diagnosis of diabetes type 1 or type 2 in the medical records before inclusion in the study are allocated to the comparison group.

\subsection{Timeline}

The first patient was included in February 2013 and the last patient was included in June 2017. Patients' data registration is planned to be completed in March 2018.

\subsection{4 | Data collection methods}

Data are obtained from hospital medical records, hospital laboratories, and official death registries. Participation in the study results in additional blood sampling and extended collection of specimens for microbiological analysis.

\subsection{5 | Registered variables}

Description of clinical variables registered in the INFECT project including principles of bacterial categorization are available in a separate publication. $^{31}$

Among patients with diagnosed diabetes, available $\mathrm{A} 1 \mathrm{C}$ values from up to 6 months before inclusion are retrospectively collected from medical records.

Also in retrospect, patients with diabetes will be categorized as type 1 , insulin-treated type 2, or non-insulin-treated type 2 based on information from medical records.

\subsection{Data management}

All research data are handled confidentially and in accordance with national laws and regulations. Patients' data are anonymized, using an individual study code. Code lists are kept locked up and separate from registered study data. Original records will be retained at trial sites for the time specified by national regulations.

A common electronical database (eCRF) with all recorded data is kept in Copenhagen, Denmark (Danish Data protection Agency approval 30-0900). The trial database will be maintained for 15 years.

\subsection{7 | Missing data}

\subsection{1 | Imputation}

We will perform complete-case analyses. If the frequency of missing data for a given analysis is more than $5 \%$, the analysis will be repeated using multiple imputation based on chained equations
(MICE). The variables included in the multiple imputation will be age, SOFA score at day 1 , the presence of hematological cancer, presence of diabetes, presence of chronic obstructive pulmonary disease (COPD) or asthma, presence of current or previous cardiovascular disease, presence of chronic kidney disease, GAS found in cultures and 90-day mortality. The variables used in specific analyses will be excluded from the imputation. We will also present unadjusted, non-imputed analyses.

\subsection{2 | SAPS II score}

If values from admission blood samples are missing, values from day 2 will be used.

\subsection{3 | SOFA score}

If values from blood samples at day 1 are missing, values from day 2 will be used. Due to the nature of eCRF, day 1 can vary from 0 to 24 hours. If day 1 is $<12$ hours, the worst value from either day 1 or day 2 is used. The SOFA score has been modified, as Glasgow Coma Scale (GCS) is not included.

\subsection{4 | Diabetes type and A1C}

If the amount of missing data exceeds $20 \%$, the specific analysis will not be performed.

\section{3 | STATISTICAL METHODS}

\section{1 | General analytical principles}

For all analyses, the level of statistical significance (2-sided) is 0.05 . $P$-values will be adjusted for multiple testing by the Benjamini-Hochberg method.

\subsection{Outcomes}

\subsection{1 | Primary outcome}

The primary outcome (all-cause mortality 90 days after inclusion) expressed as dichotomous variable (0-1) will be analyzed by logistic regression with 90 days mortality as response, having diabetes (dichotomous variable) as regressor and considering age (in years) as a covariate. Data will be presented as relative risks (RR) including $95 \%$ confidence intervals $(\mathrm{Cl})$.

\subsection{2 | Secondary outcomes}

The secondary outcomes will be analyzed by Fisher's exact test for categorical variables (amputation, affected body area, and renal replacement therapy) and by Mann-Whitney-Wilcoxon test for the continuous variables (SOFA score and SAPS II). Specific considerations are mentioned below. 
Number of days alive and out of hospital at day 90 and days alive and out of ICU at day 30 will be analyzed by Mann-WhitneyWilcoxon test.

The presence of specific bacterial species in cultures of blood or tissue expressed as dichotomous variables (0-1) will be analyzed by logistic regression considering affected body area at admission as covariates. Data will be presented as RR including $95 \% \mathrm{Cl}$.

\subsection{3 | Exploratory outcomes}

All-cause mortality at 90 days expressed as dichotomous variable (0-1) will be analyzed by logistic regression with mortality at 90 days as response and considering age (in years) as a covariate. Regressors will be either dichotomous variables (diabetes subgroups: type 1 and type 2 or insulin-treated and non-insulin-treated) or a continuous variable (A1C values before admission) depending on the outcome of interest. Data will be presented as RR including $95 \% \mathrm{Cl}$.

The exploratory outcomes investigating association with $\mathrm{A} 1 \mathrm{C}$ values before admission will be analyzed by Mann-Whitney-Wilcoxon test for continuous variables (number of days alive and out of ICU) and Fisher's exact test for categorical variables (affected body area, renal replacement therapy). Specific considerations are mentioned below.

The presence of specific bacterial species in cultures of blood or tissue expressed as dichotomous variables (0-1) will be analyzed by logistic regression considering affected body area at admission as covariates. Data will be presented as RR including $95 \% \mathrm{Cl}$.

\subsubsection{Clinical data, comorbidities, risk factors for NSTI, and medication}

Data will be presented as number/total number (percentage), median (IQR), or mean (SD).

\section{3 | Sample size estimation and sensitivity analysis}

A total of 400 patients or more are expected to be included. Estimating a diabetes incidence between $25 \%$ and $35 \%$, the group sizes will range between $N_{1}=100$ and 140 for the diabetics and $N_{2}=300$ and 260 for the nondiabetics.

Sensitivity analysis was performed with G*Power. ${ }^{33}$

With a power of 0.8 and a confidence level $\alpha=0.05$, the smallest difference in proportions estimated with a Fisher's exact test range between $7 \%$ and $12.5 \%$ both for $N_{1} / N_{2}=100 / 300$ and $N_{1} /$ $N_{2}=140 / 260$.

For differences in mean, estimated with Mann-Whitney-Wilcoxon, the smallest estimable effect is 0.29 for $N_{1} / N_{2}=100 / 300$ and 0.27 for $N_{1} / N_{2}=140 / 260$.

For odds ratios calculated by estimating logistic regression, the estimable odds ratios are either smaller than 0.49 or higher than 1.80 for $N=400$.

\section{DISCUSSION}

Diabetes is a common disease worldwide. Its impact on outcome among patients with NSTI is reported to be negative. This study will provide clinical data regarding diabetes frequency and outcome for patients with diabetes in a prospective Scandinavian NSTI cohort study. The cohort will, compared to earlier studies, be large. The prospective design of the study is a strength, yet the observational design precludes causative conclusions.

An unknown number of patients will likely be misclassified as nondiabetics. They represent individuals who, before developing NSTI, would have fulfilled the diagnostic criteria for diabetes type 2 , but had yet not received a diagnosis.

Metabolic control before hospitalization as well as type of diabetes (type 1 or 2) and treatment (insulin or non-insulin) might influence outcome in NSTI. There are numerous flaws connected to using retrospective $\mathrm{A} 1 \mathrm{C}$ values. Nothing is known of the clinical circumstances when the $\mathrm{A} 1 \mathrm{C}$ samples were collected. The time elapsed between $\mathrm{A} 1 \mathrm{C}$ sample acquisition and hospital admission will be variable among patients. This, in combination with missing $\mathrm{A} 1 \mathrm{C}$ values, makes estimation of blood glucose control before admission unreliable. Retrospective categorization of diabetes type and its treatment (insulin, non-insulin) for each included patient might be either not feasible or incorrect. Therefore, results from exploratory outcomes must be interpreted with caution.

Certain patient comorbidities, for example, peripheral vascular disease and chronic liver disease, are not clearly defined in the study protocol. This entails subjectivity and a risk of bias when registering patient data.

The use of 4 arbitrarily defined body areas to describe the infectious locus makes data compilation and statistical analysis easier. However, all categorization results in loss of information and reduced precision.

There is a relatively high number of secondary and exploratory outcomes. Statistical adjustment for multiple testing is used to reduce this risk of falsely positive findings. The amount of patients eligible for calculation of the exploratory outcomes will probably only number about 100-120, making statistical inference less precise.

Despite its limitations, this study will provide important data on patients with NSTI and diabetes.

\section{ETHICS}

The study is conducted in compliance with $\mathrm{ICH}$ Good Clinical Practice, national laws, applicable regulatory requirements and in accordance with the ethical principles in the Declaration of Helsinki. The study is approved by ethical committees in all participating countries (Danish ethical committee [1211709], Swedish ethical committee in Gothenburg [930-12], Regional committee for ethics in medical research in Western Norway [2012/2227]) 
Patients get no direct benefit from participation in the study. Hopefully results from the study will lead to improved treatment for diabetic patients with NSTI in the future.

\section{PUBLICATION}

Results will be reported in accordance with STROBE guidelines for cohort studies $^{34}$ and submitted for publication in a peer-reviewed journal.

\section{ACKNOWLEDGEMENTS}

Authors would like to thank the clinical and research personnel at each of the including hospitals, the microbiological laboratories, as well as participating patients and their relatives.

\section{CONFLICTS OF INTEREST}

The department of intensive care, Rigshospitalet, receives support for research from CSL Behring, Ferring Pharmaceuticals and Fresenius Kabi, but the authors emphasize that the support has not been used as contribution to this study. No other conflicts of interest are reported by the authors.

\section{AUTHORS' CONTRIBUTIONS}

A. N.-T. is a coordinator of the INFECT project. A. R. drafted the first manuscript. F. B., E. S., and V. M. S. revised the statistical details of the statistical analysis plan. P. A., S. S., and O. H. are principal investigators and have contributed to study design and coordinated study conduct. M. B. M. is responsible for the eCRF. A. R., P. A., M. B. M., O. H., S. S., J. T., S. A., Y. K., M. N., and G. H. have contributed to patient inclusion and data collection. All authors contributed to the writing of this manuscript and approved the final version.

\section{ORCID}

A. Rosén iD http://orcid.org/0000-0001-6739-6730

M. B. Madsen (iD http://orcid.org/0000-0003-0186-8971

\section{REFERENCES}

1. Uehara K, Yasunaga H, Morizaki Y, Horigucji H, Fushimi K, Tanaka S. Necrotising soft-tissue infections of the upper limb. Risk factors for amputation and death. Bone Joint J. 2014;96-B:1530-1534.

2. Psoinos CM, Flahive JM, Shaw JJ, et al. Contemporary trends in necrotizing soft-tissue infections in the United States. Surgery. 2013;153:819-827.

3. Hakkarainen TW, Burkette Ikebata N, Bulger E, Evans HL. Moving beyond survival as a measure of success: understanding the patient experience of necrotizing soft-tissue infections. J Surg Res. 2014;192:143-149.

4. Stevens DL, Bryant AE. Necrotizing soft-tissue infections. N Engl J Med. 2017;377:2253-2265.

5. Elliot D, Kufera JA, Myers RAM. The microbiology of necrotizing soft tissue infections. Am J Surg. 2000;179:361-366.
6. Goldstein EJC, Anaya DA, Dellinger EP. Necrotizing soft-tissue infection: diagnosis and management. Clin Infect Dis. 2007;44:705710.

7. Brink M, Arnell P, Lycke H, Rosemar A, Hagberg L. A series of severe necrotizing soft-tissue infections in a regional centre in Sweden. Acta Anaesthesiol Scand. 2014;58:882-890.

8. de Tullio D, Rossi C, Bolzon S, Scagliarini L, Occhionorelli S. Necrotizing fasciitis: a surgical emergency. Updates Surg. 2010; 62:83-87.

9. Sarani B, Strong M, Pascual J, Schwab W. Necrotizing fasciitis: current concepts and review of the literature. J Am Coll Surg. 2009;208:279-288.

10. Chennamsetty A, Khourdaji I, Burks F, Killinger KA. Contemporary diagnosis and management of Fournier's gangrene. Ther Adv Urol. 2015;7:203-215.

11. Morpurgo E, Galandiuk S. Fournier's gangrene. Surg Clin North Am. 2002;82:1213-1224.

12. Young $\mathrm{MH}$, Aronoff DM, Engleberg NC. Necrotizing fasciitis: pathogenesis and treatment. Expert Rev Anti Infect Ther. 2005;3:279294.

13. Bruun T, Kittang BR, de Hoog BJ, et al. Necrotizing soft tissue infections caused by Streptcoccus pyogenes and Streptococcus dysgalctae subsp. Equisimilis of groups $C$ and $G$ in western Norway. Clin Microbiol Infect 2013;19:E545-E550.

14. Stevens DL, Bisno AL, Chambers HF, et al. Practice guidelines for the diagnosis and management of skin and soft tissue infections: 2014 update by the infectious diseases society of America. Clin Infect Dis. 2014;59:147-159.

15. Devaney B, Frawley G, Frawley L, Pilcher DV. Necrotising soft tissue infections: the effect of hyperbaric oxygen on mortality. Anaesth Intensive Care. 2015;43:685-692.

16. Jallali N, Withey S, Butler PE. Hyperbaric oxygen as adjuvant therapy in the management of necrotizing fasciitis. Am J Surg. 2005;189:462-466.

17. Shah BJ, Hux JE. Quantifying the risk of infectious disease for people with diabetes. Diabetes Care. 2003;26:510-513.

18. Benfield T, Jensen JS, Nordestgaard BG. Influence of diabetes and hyperglycemia on infectious disease hospitalization and outcome. Diabetologia. 2007;50:549-554.

19. Kalaivani V, Bharati VH, Indumathi VA. Necrotising soft tissue infection-risk factors for mortality. J Clin Diagn Res. 2013;7:1662-1665.

20. Mills MK, Faraklas I, Davis C, Stoddard GJ, Saffle J. Outcomes from treatment of necrotizing soft-tissue infections: results from the National Surgical Quality Improvement Program database. Am J Surg. 2010;200:790-797.

21. Chen KCJ, Klingel M, McLeod S, Mindra S, Ng VK. Presentation and outcomes of necrotizing soft tissue infections. Int J Gen Med. 2017; 10:215-220.

22. Martinschek A, Evers B, Gerngross H, Schmidt R, Sparwasser C. Prognostic aspects, survival rate, and predisposing risk factors in patients with Fournier's gangrene and necrotizing soft tissue infections: evaluation of clinical outcome of 55 patients. Urol Int. 2012;89:173-179.

23. Tan JH, Koh BTH, Hong CC, et al. A comparison of necrotizing fasciitis in diabetics and non-diabetics. Bone Joint J. 2016;98-B:15631568.

24. World Health Organization. Global report on diabetes. World Health Organization; 2016. ISBN 9789241565257.

25. Dworkin MS, Westercamp MD, Park L, Mclntyre A. The epidemiology of necrotizing fasciitis including factors associated with death and amputation. Epidemiol Infect. 2009;137:1609-1614.

26. Kao LS, Knight MT, Lally KP, Mercer DW. The impact of diabetes in patients with necrotizing soft tissue infections. Surg Infect. 2005;6:427-438. 
27. Leiblein M, Marzi I, Sander AL, Barker JH, Ebert F, Frank J. Necrotizing fasciitis: treatment concepts and clinical results. Eur J Trauma Emerg Surg. 2018;44:279-290.

28. Vincent JL, de Mendonça A, Cantraine F, et al. Use of the SOFA score to assess the incidence of organ dysfunction/failure in intensive care units: results of a multicenter, prospective study. Working group on "sepsis-related problems" of the European Society of Intensive Care Medicine. Crit Care Med 1998;26:1793-1800.

29. Moreno R, Vincent JL, Matos R, et al. The use of maximum SOFA score to quantify organ dysfunction/failure in intensive care. Results of a prospective, multicenter study. Working Group on Sepsis related Problems of the ESICM. Intensive Care Med. 1999;25:686-696.

30. Le Gall JR, Lemeshow S, Saulnier F. A new Simplified Acute Physiology Score (SAPS II) based on a European/North American multicenter study. JAMA. 1993;270:2957-2963.

31. Madsen MB, Skrede S, Bruun T, et al. Necrotising soft tissue infections - a multi-centre, prospective observational study (INFECT): protocol and statistical analysis plan. Acta Anaesthesiol Scand. 2018;62:272-279.

32. INFECT-EU-FP7-HEALTH_-Systems medicine to understand severe soft tissue infections. http://www.fp7infect.eu. Accessed September 2, 2017.

33. Faul F, Erdfelder E, Buchner A, Lang AG. Statistical power analyses using G*Power 3.1: tests for correlation and regression analyses. Behav Res Methods. 2009;41:1149-1160.

34. Von Elm E, Altman DG, Egger M, Pocock SJ, Götsche PC, Vandenbroucke JP, STROBE Initiative. The Strengthening the Reporting of Observational Studies in Epidemiology (STROBE) statement: guidelines for reporting observational studies. Lancet 2007;370:1453-1457.
How to cite this article: Rosén A, Arnell P, Madsen MB, et al. Diabetes and necrotizing soft tissue infections-

A prospective observational cohort study: Statistical analysis plan. Acta Anaesthesiol Scand. 2018;62:1171-1177. https://doi.org/10.1111/aas.13130

\section{APPENDIX 1}

\section{INFECT STUDY GROUP}

Joakim Trogen, Sahlgrenska University Hospital, Gothenburg, Sweden. Sara Aronsson, Sahlgrenska University Hospital, Gothenburg, Sweden. Göran Sandström, Swedish Armed Forces, Centre for Defence Medicine, Gothenburg, Sweden. Anders Rosemar, Sahlgrenska University Hospital/Ostra, Gothenburg, Sweden. Ylva Karlsson, Blekinge County Council Hospital, Karlskrona, Sweden. Michael Nekludov, Karolinska University Hospital, Stockholm, Sweden. Gladis Helledie, Rigshospitalet, Copenhagen, Denmark. Morten Hedetoft, Rigshospitalet, Copenhagen, Denmark. Marco Bo Hansen, Rigshospitalet, Copenhagen, Denmark. Peter Polzik, Rigshospitalet, Copenhagen, Denmark. 